\title{
Variation in the Content of Cyanogenic Glycosides in Flaxseed Meal from Twenty-One Varieties
}

\author{
Roberto Russo, Remo Reggiani* \\ Istituto di Biologia e Biotecnologia Agraria, CNR, Milano, Italy \\ Email: ${ }^{*}$ reggiani@ibba.cnr.it
}

Received 26 May 2014; revised 30 June 2014; accepted 12 July 2014

Copyright (C) 2014 by authors and Scientific Research Publishing Inc.

This work is licensed under the Creative Commons Attribution International License (CC BY).

http://creativecommons.org/licenses/by/4.0/

c) (i) Open Access

\begin{abstract}
Flaxseed meal from twenty-one varieties has been evaluated for content of cyanogenic glycosides (linamarin, linustatin and neolinustatin). Varieties belonging to three different groups of productive attitude (oil, fiber and intermediate) were considered. The total content of cyanogenic glycosides ranged from 0.74 to $1.60 \mathrm{~g} \cdot \mathrm{Kg}^{-1} \mathrm{CN}^{-}$. As expected, linamarin was a minority component accounting for only $2 \%-14 \%$ of total glycosides. Linustatin resulted statistically lower in the intermediate group than that in the other groups and, in particular, it was the lowest in the Festival variety. Neolinustatin resulted lower in the fiber group although the variety Ventimiglia (belonging to oil group) showed a negligible level of this compound. Neolinustatin was positively correlated to total cyanogenic glycosides $(r=0.709)$ and inversely correlated to linustatin $(r=-0.326)$. The variability observed in the content of the various forms of glycosides could be exploited in breeding programs to reduce the content of these toxic compounds in flaxseed meal.
\end{abstract}

\section{Keywords}

High Performance Liquid Chromatography, Linamarin, Linustatin, Neolinustatin, Flaxseed Meal

\section{Introduction}

The cyanogenic glycosides (CG) are secondary plant metabolites that are composed of an $\alpha$-hydroxynitriles type aglycone and a sugar moiety (glucose or gentiobiose) [1]. These toxic molecules are synthesized from different amino acids and, through a tonoplast glycosyltransferase, accumulated in vacuoles [2] [3]. When the cell structure is destroyed by a predator, CG are rapidly decomposed to hydrogen cyanide by a two-step process catalyzed

*Corresponding author.

How to cite this paper: Russo, R. and Reggiani, R. (2014) Variation in the Content of Cyanogenic Glycosides in Flaxseed Meal from Twenty-One Varieties. Food and Nutrition Sciences, 5, 1456-1462. http://dx.doi.org/10.4236/fns.2014.515159 
by $\beta$-glucosidase and $\alpha$-hydroxynitrile lyase [1]. Cyanide is toxic to humans and animals due to its ability to link with iron, manganese or copper ions that are functional groups of many enzymes including those involved in the reduction of oxygen in the cytochrome respiratory chain. Acute intoxications are characterized by symptoms such as vomiting, nausea, headache, dizziness, difficulty in vision, hyperpnoea, arrhythmias and may finally results in collapse and death [4].

The distribution of the CG in the plant kingdom is relatively wide, including many taxa belonging to families Fabaceae, Rosaceae, Linaceae and others [1]. In an European agricultural context, the main sources of CG are seeds and by-products of crops such as flax (Linus usitatissimum), apricot (Prunus armeniaca), bitter almond (Prunus dulcis) sorghum (Sorghum vulgare) wheat (Triticum aestivum), barley (Hordeum vulgare) oat (Avena sativa) and apple (Malus pumila) [5].

Since ancient times, common flax (Linus usitatissimum L.) has been cultivated both as fiber and oilseed crop. Flaxseed fiber has a wide range of applications in various areas such as textile, paper and biocomposite manufacturing [6]. The flax as oilseed is an attractive nutrition crop, because of a high content of alpha linolenic-acid, its dietary fiber and high quality proteins. Linseed oil can be exploited both for medicinal application as antitumoral and anti-infiammatory remedy and as drying oil in paints and varnishes [7]. The remaining flour after defatting contains CG which are regarded as antinutrients. High levels of cyanogenic compounds severely restrict the amount of flax meals in animal rations [8]. The possibility of improving the commercial value of these flours by reducing the levels of CG has generated considerable interest in understanding their changes in flaxseed.

The CG in flaxseed have been identified as linustatin ( $\beta$-gentobioside of acetone cyanohydrins) that occurs also in cassava, neolinustatin ( $\beta$-gentobioside of methyl ethyl ketone cyanohydrins) present in Passiflora species and a minor component, linamarin (1-cyano-1-methylethyl ketone cyanohydrin $\beta$-D-glucopyranoside) which is present in cassava, lima bean, rubber tree, wheat and oat [8].

In this paper, we evaluated the content and distribution of individual CG in twenty-one flax varieties divided in three groups of productive attitude (oil, fiber and intermediate). The characterization quali-quantitative of CG in these varieties may prove important for a future flax breeding.

\section{Material and Methods}

\subsection{Plant Materials}

Seeds from flax varieties Valoal, Linoal, Natural, Merlin, Solal, Festival and Kaolin were obtained from Semfor (Italy). All the other varieties were kindly provided by Centro di Ricerca per le Colture Industriali, CRA (Bologna, Italy). Origin and productive attitude of the flax varieties are shown in Table 1.

\subsection{Cyanogenic Glycoside Extraction End Assay}

Whole flaxseeds were ground in a mortar and defatted by extracting the samples with hexane (1:10, w/v, twice). CG were extracted according to Russo and Reggiani [9] from defatted flour with $80 \%$ ethanol at $70^{\circ} \mathrm{C}$ twice. The alcoholic samples were dried at $70^{\circ} \mathrm{C}$ and then resuspended in water:methanol:choloroform $(25: 25: 50, \mathrm{v} / \mathrm{v})$. After $1 \mathrm{~h}$ of agitation, the samples were centrifuged for 15 minutes at $15,000 \times \mathrm{g}$ and the lower water layer recovered.

Total CG were assayed on the water extracts by the Spectroquant Cyanide-Test (Merck, Germany) according to the manufacturer's protocol.

\subsection{HPLC analysis of Cyanogenic Glycosides}

Before HPLC analysis, the samples were filtered ( $0.45 \mu$ m nylon VWR centrifugal filter, VWR-PBI, Italy $)$ and stored at $4^{\circ} \mathrm{C}$ until analysis.

For the separation of CG, we modified the method of Oomah et al. [8]. The HPLC system consisted of a Jasco PU-208Plus pump (Jasco, Japan) and a Jasco UV-975 detector (Jasco, Japan) set at $230 \mathrm{~nm}$. The analytical column was a Kinetex $4.6 \mathrm{~mm} \times 125 \mathrm{~mm}, 2.6$ micron C-18 (Phenomenex, Italy) maintained at $40^{\circ} \mathrm{C}$. Two mobile phases were used: A) $0.05 \%$ phosphoric acid in HPLC grade water; B) methanol. Phase B was maintained at $2 \%$ (v/v) for $10 \mathrm{~min}$, increased linearly to $15 \%(\mathrm{v} / \mathrm{v})$ over $6 \mathrm{~min}$, increased linearly to $100 \%(\mathrm{v} / \mathrm{v})$ over $2 \mathrm{~min}$, remained to $100 \%$ for $2 \mathrm{~min}$ and then returned to $2 \%(\mathrm{v} / \mathrm{v})$ in $2 \mathrm{~min}$ to regenerate the system. Calibration curves 
Table 1. List of flax varieties used in this study with their origin and productive attitute.

\begin{tabular}{|c|c|c|}
\hline Variety & Origin & Productive Attitude \\
\hline Ita269 & Italy & Oil \\
\hline Ecotipo Ragusano & Italy & Oil \\
\hline Claudia & Italy & Oil \\
\hline IT2421 & Italy & Oil \\
\hline Cremone & Italy & Oil \\
\hline Credo & Italy & Oil \\
\hline Ventimiglia & Italy & Oil \\
\hline Roma & Italy & Oil \\
\hline Merlin & France & Intermediate \\
\hline Solal & France & Intermediate \\
\hline Kaolin & France & Intermediate \\
\hline Natural & France & Intermediate \\
\hline Linoal & France & Intermediate \\
\hline Valoal & France & Intermediate \\
\hline Festival & France & Intermediate \\
\hline Berber & Holland & Fiber \\
\hline Belinka & Holland & Fiber \\
\hline Blu di Riga & Lithuania & Fiber \\
\hline Datcha & France & Fiber \\
\hline Cruciata & Unknown & Fiber \\
\hline Ariane & France & Fiber \\
\hline
\end{tabular}

were obtained using commercially available linamarin (Sigma-Aldrich, Italy) and purified samples of linustatin and neolinustatin. For purification, peaks were collected and CG identified by cyanide test and separation by TLC [10]. The retention times were 4.9, 8.9 and 18.8 for linamarin, linustatin and neolinustatin, respectively (Figure 1).

\subsection{Statistical Analyses}

All statistical analysis were performed by SPSS version 16.0 software. Analysis of Variance (ANOVA) was applied to establish significant differences $(\mathrm{P} \leq 0.01)$ between flax varieties and productive attitude groups in the levels of CG. Mean separation was performed using Duncan's test and referring to $\mathrm{P} \leq 0.05$ probability level. Pearson's correlations between CG species were also calculated.

\section{Results and Discussion}

In Figure 2 is shown the total CG content in flours of the twenty-one flax varieties. The total CG content is the mean of three independent extractions and is expressed as $\mathrm{g} \cdot \mathrm{Kg}^{-1} \mathrm{CN}^{-}$. In the oil group (white bars), the varieties Ita269 and Ecotipo Ragusano showed the highest CG content (1.60 and $1.48 \mathrm{~g} \cdot \mathrm{Kg}^{-1} \mathrm{CN}^{-}$respectively). The varieties more interesting are those lower in total GC content since a greater quantity of flax flour may be added in feed if the content of CG is low. In particular, they were the varieties Valoal and Festival $\left(0.86 \mathrm{and} 0.74 \mathrm{~g} \cdot \mathrm{Kg}^{-1}\right.$ $\mathrm{CN}^{-}$, respectively) within the intermediate group and varieties Datcha, Cruciata and Ariane $(0.84,0.79$ and 0.77 $\mathrm{g} \cdot \mathrm{Kg}^{-1} \mathrm{CN}^{-}$, respectively) in the fiber group. 


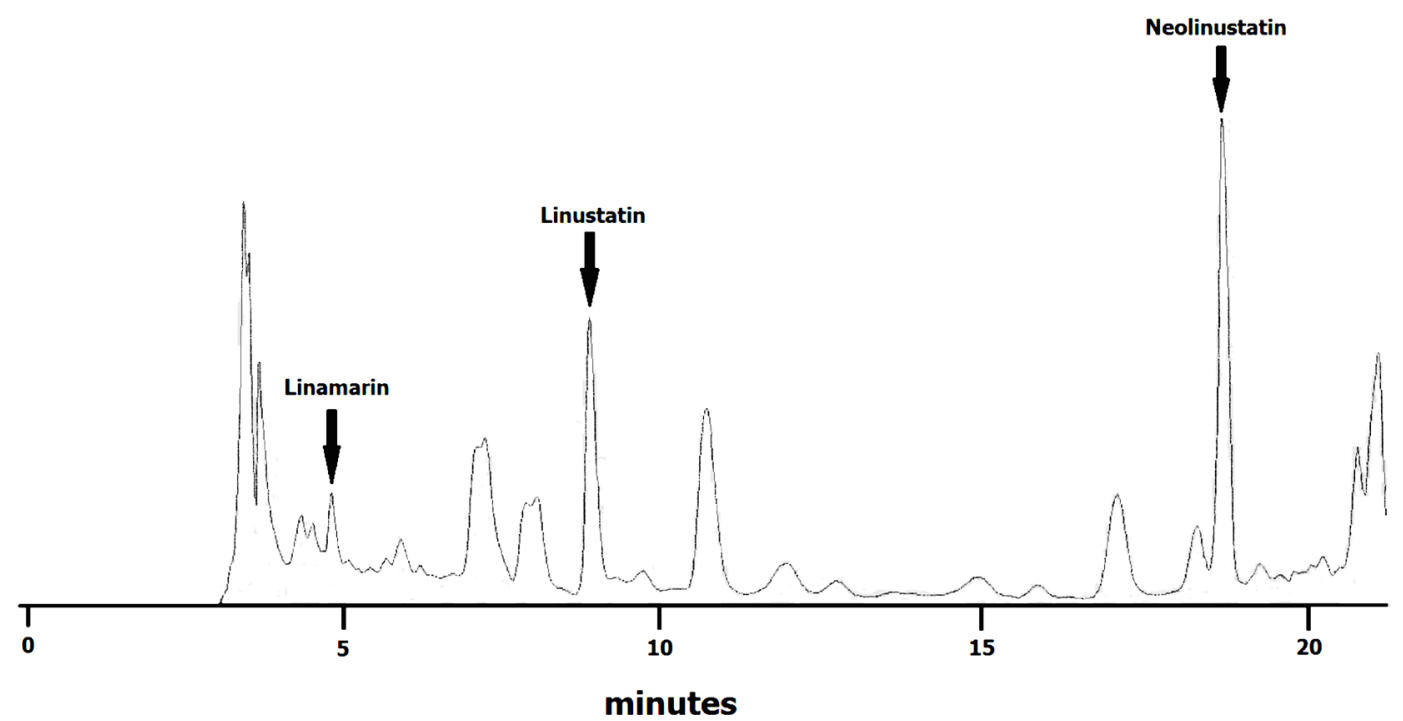

Figure 1. HPLC chromatogramme of flax cyanogenic glycosides (variety Ariane).

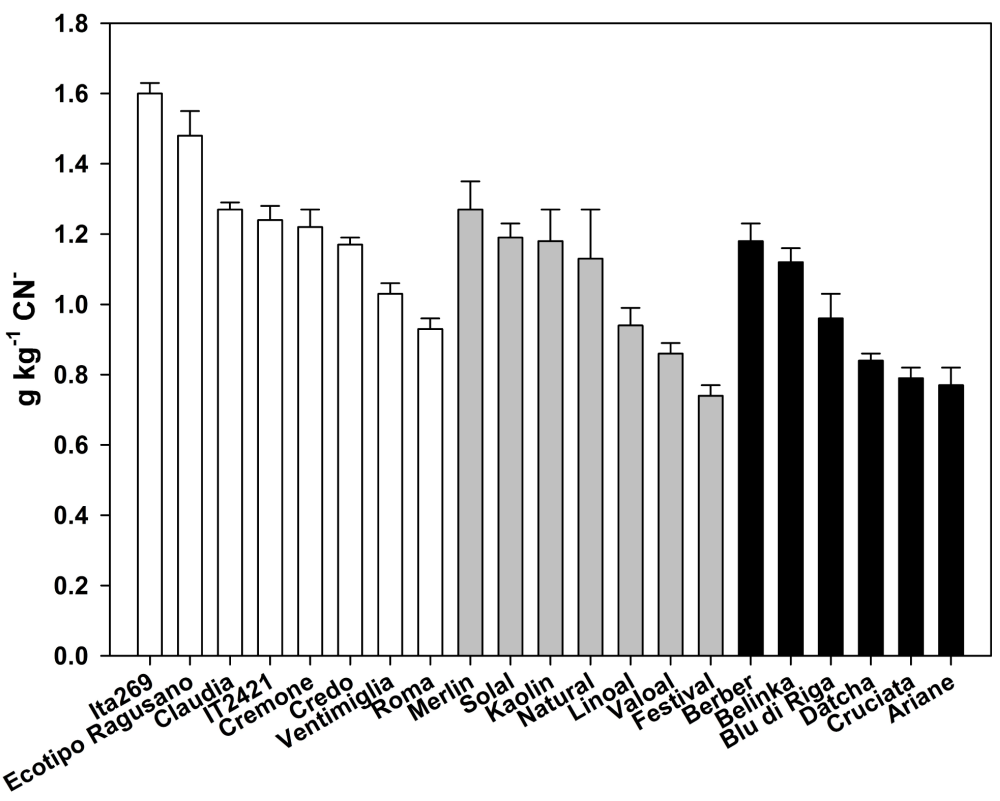

Figure 2. Cyanogenic glycoside contents in twenty-one varieties of flax divided by group of productive attitude (oil = white bars; intermediate = gray bars; fiber = black bars).

Oomah et al. [8] developed an isocratic HPLC separation for detecting CG of flax. They reported the retention times of linamarin, linustatin and neolinustatin and were 8.2, 11.0 and $23.6 \mathrm{~min}$, respectively. We modified this HPLC method by adding a mild gradient of methanol. In this way, we were able to get an acceptable separation of flax CG in less time (Figure 1).

The relative amounts of the three CG present in flax varieties are shown in Table 2. The mean of linamarin, linustatin and neolinustatin for each group of productive attitude is also shown. The ANOVA showed that the differences in the content of the three CG among varieties are significant at 0.01 level. According to Oomah et al. [8], the HPLC method found only traces of linamarin, about $5 \%-8 \%$ of the total CG in all flax varieties. From our analysis, we found a greater variability in the relative content of linamarin ( $2 \%-14 \%)$, though still was a minority component as absolute value and statistically similar among groups of productive attitude (Table 2). Instead, linustatin and neolinustatin exhibited significant differences among groups (Table 2). Linustatin re- 
sulted to be statistically lower in the flours of the intermediate group, and, in particular, should be noted the lowest level observed in the variety Festival $\left(0.28 \mathrm{~g} \cdot \mathrm{Kg}^{-1} \mathrm{CN}^{-}\right.$, letter a by the Duncan's range test). In the case of neolinustatin, the fiber group showed an average value statistically lower than the other two groups. However, it should be emphasized that the variety Ventimiglia in the oil group had a very low content of linustatin ( $0.06 \mathrm{~g}$ $\mathrm{g} \cdot \mathrm{Kg}^{-1} \mathrm{CN}^{-}$, letter a by the Duncan's range test), which it was equivalent to just $5.8 \%$ of the total of CG. No association between GC content and geographical origin was evident.

Table 3 shows the Pearson's correlation coefficients (r) between each CG compound and the total CG for the

Table 2. Linamarin, linustatin and neolinustatin contents in different flax varieties.

\begin{tabular}{|c|c|c|c|}
\hline Variety & Linamarin $^{1}$ & Linustatin $^{1}$ & Neolinustatin $^{1}$ \\
\hline Ita269 & 0.10 & $0.65(\mathrm{k})$ & $0.85(\mathrm{l})$ \\
\hline Ecotipo Ragusano & 0.14 & $0.49(\mathrm{~h})$ & 0.85 (l) \\
\hline Claudia & 0.08 & 0.53 (i) & $0.67(\mathrm{~h})$ \\
\hline IT2421 & 0.05 & 0.56 (i) & 0.63 (g) \\
\hline Cremone & 0.07 & 0.48 (h) & $0.67(h)$ \\
\hline Credo & 0.06 & $0.38(\mathrm{e})$ & $0.72(\mathrm{k})$ \\
\hline Ventimiglia & 0.02 & $0.95(\mathrm{~m})$ & 0.06 (a) \\
\hline Roma & 0.07 & 0.42 (fg) & $0.44(\mathrm{e})$ \\
\hline Mean \pm SE & $0.07 \pm 0.01$ & $0.557 \pm 0.068$ & $0.611 \pm 0.098$ \\
\hline Merlin & 0.18 & 0.53 (i) & 0.55 (f) \\
\hline Solal & 0.11 & 0.40 (ef) & 0.69 (hi) \\
\hline Kaolin & 0.11 & 0.36 (de) & 0.71 (ik) \\
\hline Natural & 0.08 & $0.38(\mathrm{e})$ & 0.67 (h) \\
\hline Linoal & 0.08 & 0.32 (bc) & 0.54 (f) \\
\hline Valoal & 0.06 & 0.46 (gh) & $0.34(\mathrm{c})$ \\
\hline Festival & 0.07 & 0.28 (a) & 0.39 (d) \\
\hline Mean \pm SE & $0.10 \pm 0.02$ & $0.389 \pm 0.035$ & $0.556 \pm 0.059$ \\
\hline Berber & 0.08 & 0.76 (l) & 0.34 (c) \\
\hline Belinka & 0.10 & $0.62(\mathrm{k})$ & 0.40 (d) \\
\hline Blu di Riga & 0.11 & 0.54 (i) & 0.30 (b) \\
\hline Datcha & 0.05 & 0.33 (bcd) & $0.46(\mathrm{e})$ \\
\hline Cruciata & 0.09 & 0.36 (cde) & 0.35 (c) \\
\hline Ariane & 0.05 & 0.32 (b) & $0.41(d)$ \\
\hline Mean \pm SE & $0.08 \pm 0.01$ & $0.489 \pm 0.081$ & $0.376 \pm 0.025$ \\
\hline P Variety & $13.90^{* *}$ & $295.73^{* *}$ & $918.13^{* *}$ \\
\hline P Group & NS & $7.19^{* *}$ & $9.66^{* *}$ \\
\hline
\end{tabular}

${ }^{1}$ Data expressed as $\mathrm{g} \cdot \mathrm{Kg}^{-1} ;{ }^{* *}$ Significant at $\leq 0.01$.

Table 3. Pearson correlation coefficients (r) for cyanogenic glycosides in twenty-one flax varieties.

\begin{tabular}{cccc}
\hline & Linustatin & Neolinustatin & Total cyanogenic glycosides \\
\hline Linamarin & -0.082 & $0.385^{* *}$ & $0.454^{* *}$ \\
Linustatin & 1 & $-0.326^{* *}$ & $0.418^{* *}$ \\
Neolinustatin & & 1 & $0.709^{* *}$ \\
Total cyanogenic glycosides & & & 1 \\
\hline
\end{tabular}

${ }^{* *}$ Correlation is significant at the 0.01 level. 
twenty-one flax varieties considered in this study. The total CG content was positively correlated with all the glycosides, even if the correlation value significantly greater observed for neolinustatin $(r=0.709)$ could indicate that this compound is the main component of the total glycosides of flax. Moreover, the Pearson analysis showed that neolinustatin was positively correlated to linamarin $(r=0.385)$ and negatively correlated to linustatin $(r=-0.326)$. The inverse correlation between linustatin and neolinustatin might suggest a competition in the synthesis of these two compounds. However, since these two molecules are derived from different amino acids (linustatin from valine, neolinustatin from isoleucine) [11] [12], the contents of these two glycosides could be controlled by the relative levels of the two amino acids. Alternatively, the competition between the two CG may lie in the two biosynthetic pathways since the enzymes involved are common [12].

\section{Conclusion}

Flax is a plant of considerable economic importance for fiber, oil and meal. However, the CG in linseeds are the main toxic compounds that reduce its use in animal feed [9]. Decreasing the content of CG in flax meal could increase the amount of flour addition in feed (currently goes up to $12 \%-14 \%$ in ruminants [13]. We found a significant variability among flax varieties in the content of both total CG and the three different molecular species of glycosides (Figure 2, Table 2). This variability could be exploited in breeding programs to reduce the content of linustatin and neolinustatin in flaxseed. Some interesting varieties have already emerged from this study. The variety Ventimiglia showed a negligible level of neolinustatin while the variety Festival as well as presenting low levels of total CG also had the lowest content of linustatin. However, it will be necessary to verify the possibility of reducing the content of CG in suitably crosses due to the negative correlation found in this study between linustatin and neolinustatin (Table 3).

\section{Acknowledgements}

The authors thank Regione Lombardia for financial support through project “FilAgro” (No. 18093).

\section{References}

[1] Vetter, J. (2000) Plant Cyanogenic Glycosides. Toxicon, 38, 11-36. http://dx.doi.org/10.1016/S0041-0101(99)00128-2

[2] Saunders, J.A. and Conn, E.E. (1978) Presence of the Cyanogenic Glucoside Dhurrin in Isolated Vacuoles from Sorghum. Plant Physiology, 61, 154-157. http://dx.doi.org/10.1104/pp.61.2.154

[3] Gruhnert, C., Biehl, B. and Selmar, D. (1994) Compartmentation of Cyanogenic Glucosides and Their Degrading Enzymes. Planta, 195, 36-42. http://dx.doi.org/10.1007/BF00206289

[4] EFSA (European Food Safety Authority) (2007) Opinion of the Scientific Panel on Contaminats in the Food Chain on a Request from the Commision Related to Cyanogenic Compounds as Undesirable Substances in Animal Feed. EFSA Journal, 434, 1-67.

[5] EC (European Commision) (2003) Opinion of the Scientific Committee on Animal Nutrition on Undesirable Substances in Feed. European Commision, Health and Consumer Protection Directorate General, Brussels.

[6] Alix, S., Marais, S., Morvan, C. and Lebrum, L. (2008) Biocomposite Materials from Flax Plants: Preparation and Properties. Compos Part A, 39, 1793-1801. http://dx.doi.org/10.1016/j.compositesa.2008.08.008

[7] Rabetafika, H.N., Van Remoortel, V., Danthine, S., Paquot, M. and Blecker, C. (2011) Flaxseed Proteins: Food Uses and Health Benefits. International Journal of Food Science and Technology, 46, 221-228. http://dx.doi.org/10.1111/j.1365-2621.2010.02477.x

[8] Oomah, B.D., Mazza, G. and Kenaschuk E.O. (1992) Cyanogenic Compounds in Flaxseed. Journal of Agricultural and Food Chemistry, 40, 1346-1348. http://dx.doi.org/10.1021/jf00020a010

[9] Russo, R. and Reggiani, R. (2013) Variability of Antinutritive Compounds in Linus usitatissimum Flours. International Journal of Plant Biology, 4, 11-13. http://dx.doi.org/10.4081/pb.2013.e3

[10] Amarowicz, R., Chong, X. and Shahidi, F. (1993) Chromatographic Techniques for Preparation of Linustatin and Neolinustatin from Flaxseed: Standards for Glycoside Analyses. Food Chemistry, 48, 99-101. http://dx.doi.org/10.1016/0308-8146(93)90228-8

[11] Krech, M.J. and Fieldes, M.A. (2003) Analysis of the Developmental Regulation of the Cyanogenic Compounds in Seedlings of Two Lines of Linus usitatissimum. Canadian Journal of Botany, 81, 1029-1038.

http://dx.doi.org/10.1139/b03-097 
[12] Selmar, D. (2010) Biosynthesis of Cyanogenic Glycosides, Glucosinolates, and Non-Protein Amino Acids. Annual Plant Reviews, 40, 92-181.

[13] Maddock, T.D., Anderson, V.L. and Lardy, G.P. (2005) Using Flax in Livestock Diets. North Dakota Extension Service, North Dakota Agricultural Experimental Station. http://www.agmrc.org/media/cms/as1283 E9EE897D14437.pdf 
Scientific Research Publishing (SCIRP) is one of the largest Open Access journal publishers. It is currently publishing more than 200 open access, online, peer-reviewed journals covering a wide range of academic disciplines. SCIRP serves the worldwide academic communities and contributes to the progress and application of science with its publication.

Other selected journals from SCIRP are listed as below. Submit your manuscript to us via either submit@scirp.org or Online Submission Portal.
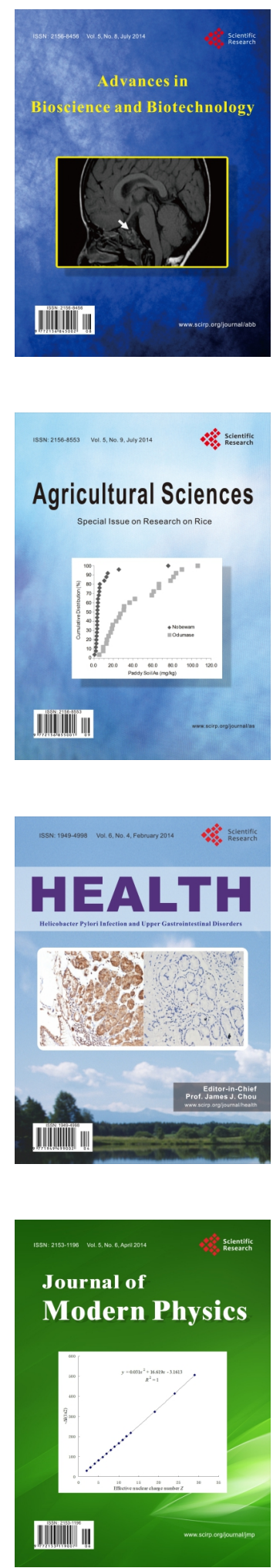
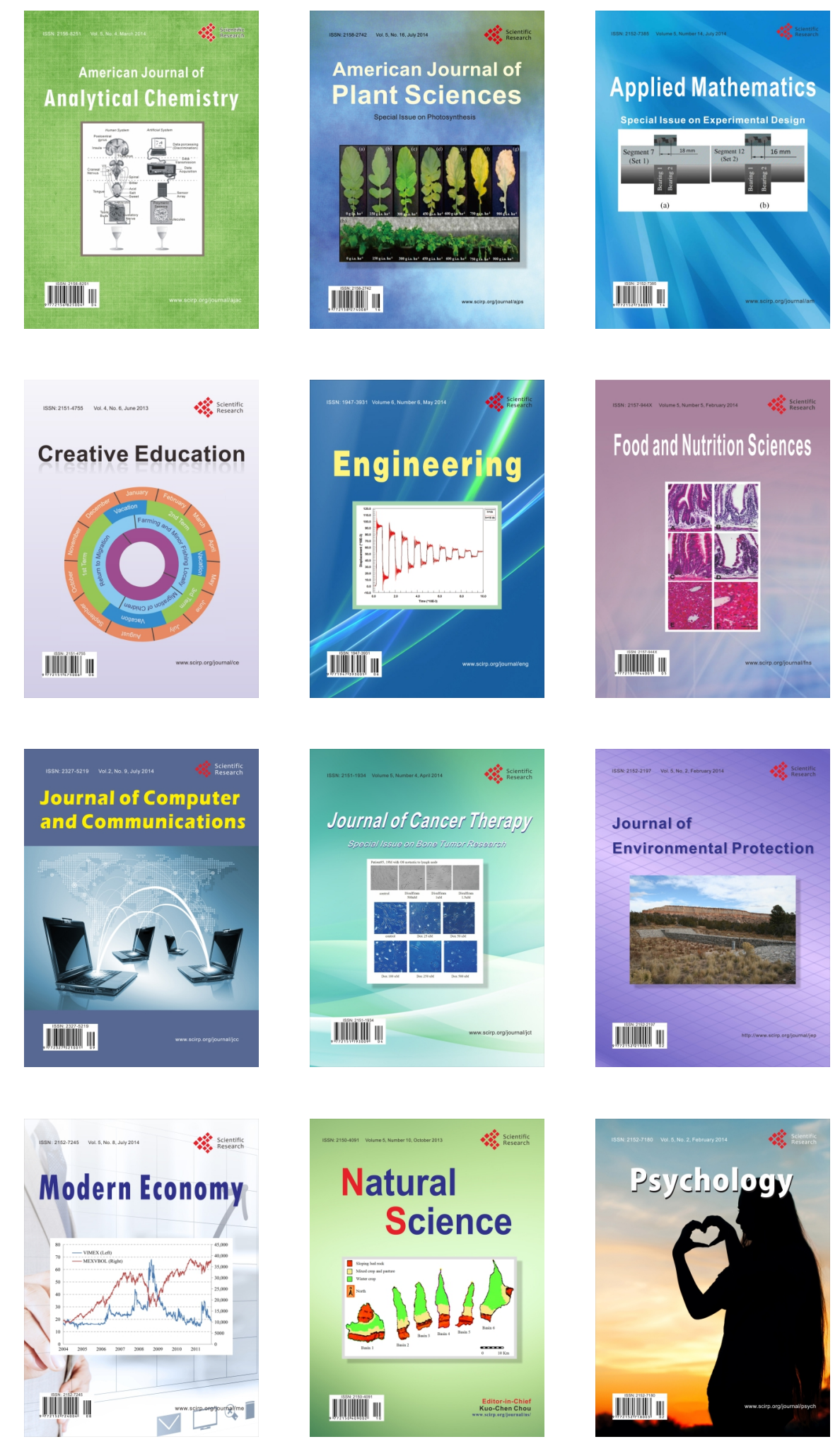